\title{
A NOTE ON THE LEAST EIGENVALUE OF A GRAPH WITH GIVEN MAXIMUM DEGREE*
}

\author{
BAO-XUAN ZHU ${ }^{\dagger}$
}

\begin{abstract}
This note investigates the least eigenvalues of connected graphs with $n$ vertices and maximum degree $\Delta$, and characterizes the unique graph whose least eigenvalue achieves the minimum among all the connected graphs with $n$ vertices and maximum vertex degree $\Delta>\frac{n}{2}$.
\end{abstract}

Key words. Maximum vertex degree, Least eigenvalue, Spectral radius.

AMS subject classifications. 05C50, 15A18.

1. Introduction. Throughout this paper all graphs are finite and simple. Graph theoretical terms used but not defined follow 3 .

Let $G=(V(G), E(G))$ be a graph with $n$ vertices. By $G-U$ we mean the induced subgraph $G[V-U]$ if $U \subset V(G)$. Denote by $N_{G}(v)$ (or $N(v)$ for short) the set of all neighbors of $v$ in $G$. The adjacency matrix of $G$ is $A(G)=\left(a_{i j}\right)_{n \times n}$, where $a_{i j}=1$ if two vertices $v_{i}$ and $v_{j}$ are adjacent in $G$ and $a_{i j}=0$ otherwise. All eigenvalues of $A(G)$ are real and can be arranged in order as $\lambda_{1}(G) \geq \lambda_{2}(G) \geq \cdots \geq \lambda_{n}(G)$ since $A(G)$ is a real symmetric matrix. The largest eigenvalue $\lambda_{1}(G)$ of $A(G)$ is called the spectral radius of $G$, denoted by $\rho(G)$. The least eigenvalue $\lambda_{n}(G)$ is also denoted by $\lambda_{\min }(G)$. Assume that $x=\left(x_{v_{1}}, x_{v_{2}}, \ldots, x_{v_{n}}\right)^{T} \in \mathbb{R}^{n}$ and $x$ is a unit eigenvector of $A(G)$ corresponding to $\lambda_{\min }(G)$. Then by the Rayleigh-Ritz Theorem, we have

$$
\lambda_{\min }(G)=\min _{\substack{y \in \mathbb{R}^{n} \\\|y\|=1}} y^{T} A(G) y=x^{T} A(G) x=2 \sum_{v_{i} v_{j} \in E(G)} x_{v_{i}} x_{v_{j}}
$$

and

$$
\lambda_{\min }(G) x_{v}=\sum_{u \in N_{G}(v)} x_{u}, \text { for each } \mathrm{v} \in V(G) .
$$

The research for the least eigenvalue of graphs in some class is well-studied and interesting. For example, Bell et al. [1, 2] studied the extremal graphs with $n$ vertices

\footnotetext{
*Received by the editors on March 4, 2011. Accepted for publication on May 19, 2012. Handling Editor: Bryan L. Shader.

${ }^{\dagger}$ School of Mathematical Sciences, Jiangsu Normal University, Xuzhou 221116, P.R. China (zhubaoxuan@yahoo.com.cn). This work was supported partially by the NSF of of Xuzhou Normal University (no. 11XLR30).
} 
and $m$ edges having the minimal least eigenvalues. Constantine [5] showed that

$$
\lambda_{\min }(G) \geq-\sqrt{\left\lfloor\frac{n}{2}\right\rfloor\left\lceil\frac{n}{2}\right\rceil}
$$

for any graph of order $n$, where equality holds if and only if $G=K_{\left\lfloor\frac{n}{2}\right\rfloor,\left\lceil\frac{n}{2}\right\rceil}$. Cioaba, Gregory, and Nikiforov [4] also proved that if $G$ is a graph of order $n$ with diameter $D$ and maximum vertex degree $\Delta$, then

$$
\lambda_{\min }(G) \geq-\Delta+\frac{1}{n(D+1)} .
$$

Fan et al. 7] obtained unicyclic graphs having the minimal least eigenvalues. Liu et al. 8] determined the graph with the minimal least eigenvalue among all unicyclic graphs with a given number of pendant vertices. Petrovic et al. 9] obtained the bicyclic graph minimizing the least eigenvalue. Wang and Fan [10] gave the graph of order $n$ with $k$ cut vertices having the minimal least eigenvalue. Ye et al. [11] discussed the least eigenvalues of graphs with given connectivity. Zhu [12] also consider the least eigenvalues of graphs with cut vertices or edges.

In this note, we consider the following problem: what is the structure of the graph having the minimal least eigenvalue among all connected graphs with $n$ vertices and maximum vertex degree $\Delta$ ?

Let the complete bipartite graph $K_{p, q}$ have the vertex bipartition $\left(V_{1}, V_{2}\right)$, where $V_{1}=\left\{v_{1}, v_{2}, \ldots, v_{p}\right\}$ and $V_{2}=\left\{v_{p+1}, \ldots, v_{p+q}\right\}$. Let the graph $H_{p, q}^{s, t}$ be obtained from $K_{p, q}$ by adding a new vertex $u$ and joining $u$ to $s$ vertices of $V_{1}$ and $t$ vertices of $V_{2}$.

From the above result of Constantine, we know that $\lambda_{\min }(G) \geq-\sqrt{\left\lfloor\frac{n}{2}\right\rfloor\left\lceil\frac{n}{2}\right\rceil}$ for any connected graph $G$ with $n$ vertices and maximum vertex degree $\Delta=\left\lceil\frac{n}{2}\right\rceil$, where equality holds if and only if $G=K_{\left\lfloor\frac{n}{2}\right\rfloor,\left\lceil\frac{n}{2}\right\rceil}$. The main result of this note is the following:

THEOREM 1.1. Of all the connected graphs of order $n$ with given maximum degree $\Delta>\left\lceil\frac{n}{2}\right\rceil$, (i) the graph with the minimal least eigenvalue is isomorphic to $H_{\left\lceil\frac{n-1}{2}\right\rceil+2,\left\lfloor\frac{n-1}{2}\right\rfloor-2}^{\left\lceil\frac{n-1}{2}\right\rceil+2,-2}$ for $\Delta=\left\lceil\frac{n}{2}\right\rceil+1$ and $n \geq 5$; (ii) the graph with the minimal least eigenvalue is isomorphic to either $H_{\left\lceil\frac{n-1}{2}\right\rceil,\left\lfloor\frac{n-1}{2}\right\rfloor}^{\left\lceil\frac{n-1}{2}\right\rceil, \Delta-\left\lceil\frac{n-1}{2}\right\rceil}$ or $H_{\left\lceil\frac{n-1}{2}\right\rceil+1,\left\lfloor\frac{n-1}{2}\right\rfloor-1}^{\left\lceil\frac{n-1}{2}\right\rceil+1, \Delta-\left\lceil\frac{n-1}{2}\right\rceil-1}$ for $\Delta>\left\lceil\frac{n}{2}\right\rceil+1$.

REMARK 1.2. From the proof of Theorem 1.1. we know that $\lambda_{\min }(G)>$ $-\sqrt{\left\lceil\frac{n}{2}\right\rceil\left\lfloor\frac{n}{2}\right\rfloor}$ if $G$ is a connected graph of order $n$ with maximum degree $\Delta>\left\lceil\frac{n}{2}\right\rceil$. Thus,

$$
\lambda_{\min }(G)>-\sqrt{\left\lceil\frac{n}{2}\right\rceil\left\lfloor\frac{n}{2}\right\rfloor}>-\Delta+\frac{1}{n(D+1)} .
$$


This improves the bound in Eq. (1.3).

By this theorem, we further obtain the next result.

Corollary 1.3. Let $G$ be be a connected graph of order $n$ with given maximum degree $\Delta>\left\lceil\frac{n}{2}\right\rceil+1$.

(i) If $n$ is even for $\left\lceil\frac{n}{2}\right\rceil+1<\Delta \leq \frac{3 n-2-\sqrt{3 n^{2}-12 n}}{2}$ or $n$ is odd for $\left\lceil\frac{n}{2}\right\rceil+1<\Delta \leq$ $\frac{3 n-2-\sqrt{2 n^{2}-4 n+3}}{2}$, then we have

$$
\lambda_{\min }(G) \geq \lambda_{\min }\left(H_{\left\lceil\frac{n-1}{2}\right\rceil+1,\left\lfloor\frac{n-1}{2}\right\rfloor-1}^{\left\lceil\frac{n-1}{2}\right\rceil+1, \Delta-\left\lceil\frac{n-1}{2}\right\rceil-1}\right)
$$

with equality if and only if $G \cong H_{\left\lceil\frac{n-1}{2}\right\rceil+1,\left\lfloor\frac{n-1}{2}\right\rfloor-1}^{\left\lceil\frac{n-1}{2}\right\rceil+1, \Delta-\lceil\text {. }}$.

(ii) If $n$ is even for $\frac{3 n-\sqrt{3 n^{2}-8 n+4}}{2} \leq \Delta \leq n-1$ or $n$ is odd for $\frac{3 n-2-\sqrt{2 n^{2}-6 n+5}}{2} \leq$ $\Delta \leq n-1$, then we have

$$
\lambda_{\min }(G) \geq \lambda_{\min }\left(H_{\left\lceil\frac{n-1}{2}\right\rceil,\left\lfloor\frac{n-1}{2}\right\rfloor}^{\left\lceil\frac{n-1}{2}\right\rceil, \Delta-\left\lceil\frac{n-1}{2}\right\rceil}\right)
$$

with equality if and only if $G \cong H_{\left\lceil\frac{n-1}{2}\right\rceil,\left\lfloor\frac{n-1}{2}\right\rfloor}^{\left\lceil\frac{n-1}{2}\right\rceil}$.

REMARK 1.4. In general, it is hard to characterize the corresponding extremal graphs having the minimal least eigenvalue over all the connected graphs of order $n$ with given maximum degree $\Delta<\frac{n}{2}$. It is well known that $\rho(G) \leq \Delta$ with equality if and only if $G$ is regular, and $G$ is bipartite if and only if $\rho(G)=-\lambda_{\min }(G)$ (see [6]). Thus, if $G$ is a connected graph of order $n$ with maximum degree $\Delta<\frac{n}{2}$, then $\lambda_{\min }(G) \geq-\Delta$ with equality if and only if $G$ is a regular bipartite graph.

2. Proof of Theorem 1.1. Let $G$ be a connected graph of order $n$ with given maximum vertex degree $\Delta>\left\lceil\frac{n}{2}\right\rceil$. Assume that $\lambda_{\min }(G)$ is as small as possible and that $x=\left(x_{1}, x_{2}, \ldots, x_{n}\right)^{T}$ is a unit eigenvector of $A(G)$ corresponding to $\lambda_{\min }(G)$. Denote by $V^{+}=\left\{v \in V(G): x_{v}>0\right\}, V^{-}=\left\{v \in V(G): x_{v}<0\right\}$ and $V^{0}=\{v \in$ $\left.V(G): x_{v}=0\right\}$. We will divide the following proof into two cases.

Case 1. If $\left|V^{0}\right|=0$, then we have $n-\Delta \leq\left|V^{-}\right|,\left|V^{+}\right| \leq \Delta$. Otherwise, we have

$$
\begin{aligned}
\lambda_{\min }(G) & =2 \sum_{v_{i} v_{j} \in E(G)} x_{v_{i}} x_{v_{j}} \\
& \geq 2 \sum_{v_{i} \in V^{-}, v_{j} \in V^{+}} x_{v_{i}} x_{v_{j}} \\
& \geq \lambda_{\min }\left(K_{\left|V^{-}\right|,\left|V^{+}\right|}\right) \\
& =-\sqrt{\left|V^{-}\right|\left|V^{+}\right|} \\
& >-\sqrt{\Delta(n-\Delta)} \\
& =\lambda_{\min }\left(K_{\Delta, n-\Delta}\right),
\end{aligned}
$$


a contradiction. On the other hand, if $\left\{\left|V^{-}\right|,\left|V^{+}\right|\right\}=\{n-\Delta, \Delta\}$, then every vertex in $V^{+}$must be adjacent to all vertices in $V^{-}$by Eq. (1.1) since $\lambda_{\min }(G)$ is as small as possible, which implies that $G=K_{n-\Delta, \Delta}$. Thus, in what follows we only need to consider the case for $n-\Delta+1 \leq\left|V^{-}\right|,\left|V^{+}\right| \leq \Delta-1$.

Let $u$ be a vertex with maximum degree. Without loss of generality, we can assume that $x_{u}>0$. Consequently, we obtain that every vertex in $V^{+}$must be adjacent to all vertices in $V^{-}$; otherwise there exists two disjunct vertices $u_{1} \in V^{+}$ and $v_{1} \in V^{-}$, and then by Eq. (1.1), we have a graph $G+u_{1} v_{1}$ with maximum degree $\Delta$ and

$$
\begin{aligned}
\lambda_{\min }(G) & =2 \sum_{v_{i} v_{j} \in E(G)} x_{v_{i}} x_{v_{j}} \\
& >2 \sum_{v_{i} v_{j} \in E(G)} x_{v_{i}} x_{v_{j}}+2 x_{u_{1}} x_{v_{1}} \\
& \geq \lambda_{\min }\left(G+u_{1} v_{1}\right)
\end{aligned}
$$

since $G$ is connected, which contradicts that $\lambda_{\min }(G)$ is as small as possible. Thus, we can write $G$ as $H_{s, n-s-1}^{s, \Delta-s}$ if we assume that $\left|V^{-}\right|=s$ and $\left|V^{+}\right|=n-s$. By Eq. (1.2), $x$ has a constant value on the vertices of $V^{-}$(respectively, $V^{+} \bigcap N(u)$ and $V^{+} \backslash N(u)$ ), denoted by $x_{1}$ (respectively, $x_{2}$ and $x_{3}$ ), e.g., $\lambda_{\min }(G) x_{v_{1}}=\sum_{t \in V^{+}} x_{t}=\lambda_{\min }(G) x_{v_{2}}$ for any two vertices $v_{1}, v_{2} \in V^{-}$, which implies that $x_{v_{1}}=x_{v_{2}}$ since $\lambda_{\min }(G)<0$. Hence, by Eq. (1.2), we have the following equations

$$
\begin{aligned}
& \lambda_{\min }(G) x_{u}=s x_{1}+(\Delta-s) x_{2}, \\
& \lambda_{\min }(G) x_{1}=x_{u}+(\Delta-s) x_{2}+(n-\Delta-1) x_{3}, \\
& \lambda_{\min }(G) x_{2}=s x_{1}+x_{u}, \\
& \lambda_{\min }(G) x_{3}=s x_{1} .
\end{aligned}
$$

This implies that $\lambda_{\min }(G)$ is the least root of the following equation in $\lambda$ :

$$
\lambda^{4}-[s(n-1-s)+\Delta] \lambda^{2}+(\Delta-s) s(n-1-\Delta)-2(\Delta-s) s \lambda=0 .
$$

From Eq. (2.1), we know that $\lambda_{\min }\left(H_{\left\lceil\frac{n-1}{2}\right\rceil,\left\lfloor\frac{n-1}{2}\right\rfloor}^{\left\lceil\frac{n-1}{2}\right\rceil, \Delta-\left\lceil\frac{n-1}{2}\right\rceil}\right)$ is the least root of the following equation:

$$
\lambda^{4}-\left(\left\lceil\frac{n-1}{2}\right\rceil\left\lfloor\frac{n-1}{2}\right\rfloor+\Delta\right) \lambda^{2}+\left(\Delta-\left\lceil\frac{n-1}{2}\right\rceil\right)\left\lceil\frac{n-1}{2}\right\rceil(n-1-\Delta-2 \lambda)=0 .
$$

Note that

$$
\lambda^{4}-\left(\left\lceil\frac{n-1}{2}\right\rceil\left\lfloor\frac{n-1}{2}\right\rfloor+\Delta\right) \lambda^{2}+\left(\Delta-\left\lceil\frac{n-1}{2}\right\rceil\right)\left\lceil\frac{n-1}{2}\right\rceil(n-1-\Delta-2 \lambda) \leq 0
$$


for $\lambda=-\sqrt{\left\lceil\frac{n-1}{2}\right\rceil\left\lfloor\frac{n-1}{2}\right\rfloor}$, where equality holds if and only if $\Delta=n-1$ is even, which implies that

$$
\lambda_{\min }(G) \leq \lambda_{\min }\left(H_{\left\lceil\frac{n-1}{2}\right\rceil,\left\lfloor\frac{n-1}{2}\right\rfloor}^{\left\lceil\frac{n-1}{2}\right\rceil, \Delta-\left\lceil\frac{n-1}{2}\right\rceil}\right) \leq-\sqrt{\left\lceil\frac{n-1}{2}\right\rceil\left\lfloor\frac{n-1}{2}\right\rfloor},
$$

where equality in the second inequality holds if and only if $\Delta=n-1$ is even.

Hence, we have $-\sqrt{\left\lceil\frac{n}{2}\right\rceil\left\lfloor\frac{n}{2}\right\rfloor}<\lambda_{\min }(G) \leq-\sqrt{\left\lceil\frac{n-1}{2}\right\rceil\left\lfloor\frac{n-1}{2}\right\rfloor}$, which can be seen from the result of Constantine and inequalities in $(2.2)$. Note that $-\sqrt{\Delta(n-\Delta)}$ is also the least root of the above Eq. (2.1) for $s=\Delta$. Thus, in the following we will consider the least root of above Eq. (2.1) for $n-\Delta+1 \leq s \leq \Delta$ since $\lambda_{\min }(G)=-\sqrt{\Delta(n-\Delta)}$ for $\left\{\left|V^{-}\right|,\left|V^{+}\right|\right\}=\{n-\Delta, \Delta\}$.

Assume that $f(s)=\lambda^{4}-[s(n-1-s)+\Delta] \lambda^{2}+(\Delta-s) s(n-1-\Delta)-2(\Delta-s) s \lambda$. Then $f(s)=\left[(\lambda+1)^{2}-n+\Delta\right] s^{2}-\left[(n-1) \lambda^{2}+2 \lambda \Delta-\Delta(n-1-\Delta)\right] s+\lambda^{4}-\Delta \lambda^{2}$ is a quadratic function in $s$, whose axis of symmetry is

$$
\begin{aligned}
s_{0} & =\frac{(n-1) \lambda^{2}+2 \lambda \Delta-\Delta(n-1-\Delta)}{2\left[(\lambda+1)^{2}-n+\Delta\right]} \\
& =\frac{n-1}{2}+\frac{(n-1-\Delta)(n-1-\Delta-2 \lambda)}{2\left[(\lambda+1)^{2}-n+\Delta\right]} .
\end{aligned}
$$

To consider the minimal least root of Eq. (2.1) for $n-\Delta+1 \leq s \leq \Delta$, we only need to know when $f(s)$ attains its minimum. We need the following two claims.

Claim 2.1. Let $-\sqrt{\left\lceil\frac{n}{2}\right\rceil\left\lfloor\frac{n}{2}\right\rfloor}<\lambda \leq-\sqrt{\left\lceil\frac{n-1}{2}\right\rceil\left\lfloor\frac{n-1}{2}\right\rfloor}$ and $\left\lceil\frac{n}{2}\right\rceil+1<\frac{n+4}{2} \leq \Delta$. Then we have $(\lambda+1)^{2}-n+\Delta>0$ and

$$
0 \leq \frac{(n-1-\Delta)(n-1-\Delta-2 \lambda)}{2\left[(\lambda+1)^{2}-n+\Delta\right]}<\frac{3}{2},
$$

where $(n-1-\Delta)(n-1-\Delta-2 \lambda)=0$ if and only if $\Delta=n-1$.

Proof. In fact, it is clear for the lower bound, and the upper bound is equivalent to

$$
-\Delta^{2}+(2 n+1-2 \lambda) \Delta-n^{2}-n+2 n \lambda+3 \lambda^{2}+4 \lambda+2>0 .
$$

It suffices to show that

$$
\begin{aligned}
& -\frac{(n+4)^{2}}{4}+\frac{(2 n+1-2 \lambda)(n+4)}{2}-n^{2}-n+2 n \lambda+3 \lambda^{2}+4 \lambda+2 \\
& =\frac{3 n}{2}-\frac{n^{2}}{4}+n \lambda+3 \lambda^{2} \\
& >0,
\end{aligned}
$$


which is trivial for $-\sqrt{\left\lceil\frac{n}{2}\right\rceil\left\lfloor\frac{n}{2}\right\rfloor} \leq \lambda \leq-\sqrt{\left\lceil\frac{n-1}{2}\right\rceil\left\lfloor\frac{n-1}{2}\right\rfloor}$.

Similarly, we can easily prove the next claim.

Claim 2.2. Let $-\sqrt{\left\lceil\frac{n}{2}\right\rceil\left\lfloor\frac{n}{2}\right\rfloor}<\lambda \leq-\sqrt{\left\lceil\frac{n-1}{2}\right\rceil\left\lfloor\frac{n-1}{2}\right\rfloor}, \Delta=\left\lceil\frac{n}{2}\right\rceil+1$ and $n \geq 5$. Then we have

$$
\left\lceil\frac{n-1}{2}\right\rceil+2<\frac{n-1}{2}+\frac{(n-1-\Delta)(n-1-\Delta-2 \lambda)}{2\left[(\lambda+1)^{2}-n+\Delta\right]}<\left\lceil\frac{n-1}{2}\right\rceil+\frac{5}{2} .
$$

Thus, by virtue of Claim 2.1, $f(s)$ can attain the minimum value only at $s=$ $\left\lceil\frac{n-1}{2}\right\rceil$ or $\left\lceil\frac{n-1}{2}\right\rceil+1$ for $\left\lceil\frac{n}{2}\right\rceil+2 \leq \Delta<n-1$, and $f(s)$ can attain the minimum $f\left(\left\lceil\frac{n-1}{2}\right\rceil\right)=f\left(\left\lfloor\frac{n-1}{2}\right\rfloor\right)$ for $\Delta=n-1$ if and only if $s=\left\lceil\frac{n-1}{2}\right\rceil$ or $s=\left\lfloor\frac{n-1}{2}\right\rfloor$. It follows that if $\Delta>\left\lceil\frac{n}{2}\right\rceil+1$ then the graph $G$ with the minimal least eigenvalue is isomorphic to $H_{\left\lceil\frac{n-1}{2}\right\rceil,\left\lfloor\frac{n-1}{2}\right\rfloor}^{\left\lceil\frac{n-1}{2}\right\rceil, \Delta-\left\lceil\frac{n-1}{2}\right\rceil}$ or $H_{\left\lceil\frac{n-1}{2}\right\rceil+1,\left\lfloor\frac{n-1}{2}\right\rfloor-1}^{\left\lceil\frac{n-1}{2}\right\rceil+1, \Delta-\left\lceil\frac{n-1}{2}\right\rceil-1}$. In addition, in view of Claim 2.2, $f(s)$ can attain the minimum value only at $s=\left\lceil\frac{n-1}{2}\right\rceil+2$ for $\Delta=\left\lceil\frac{n}{2}\right\rceil+1$ and $n \geq 5$. This completes the proof of the Case 1.

Case 2. If $\left|V^{0}\right| \neq 0$, then let $y$ be a subvector of $x$ by deleting the entries corresponding to $v \in V^{0}$. By Eq. (1.1), we have

$$
\begin{aligned}
\lambda_{\min }(G) & =2 \sum_{v_{i} v_{j} \in E(G)} x_{v_{i}} x_{v_{j}} \\
& =y^{T} A\left(G-V^{0}\right) y \\
& \geq \lambda_{\min }\left(G-V^{0}\right) \\
& \geq-\sqrt{\left\lfloor\frac{n-1}{2}\right\rfloor\left\lceil\frac{n-1}{2}\right\rceil},
\end{aligned}
$$

where the equality in the first inequality holds if and only if $y$ is a least vector of $G-V^{0}$, and the equality in the second inequality holds if and only if $G-V^{0} \cong K_{\left\lceil\frac{n-1}{2}\right\rceil,\left\lfloor\frac{n-1}{2}\right\rfloor}$. Thus, if $\left|V^{0}\right| \neq 0$, then we have

$$
\lambda_{\min }(G) \geq-\sqrt{\left\lfloor\frac{n-1}{2}\right\rfloor\left\lceil\frac{n-1}{2}\right\rceil}
$$

with equality only when $V^{0}=\{u\}$ and $G=H_{\left\lceil\frac{n-1}{2}\right\rceil,\left\lfloor\frac{n-1}{2}\right\rfloor}^{b, \Delta-b}$ for some positive integer $b$. This completes the proof of the Case 2 .

Finally, if the extremal graph $G$ belongs to Case 2, then

$$
\lambda_{\min }(G) \geq-\sqrt{\left\lfloor\frac{n-1}{2}\right\rfloor\left\lceil\frac{n-1}{2}\right\rceil}
$$


with equality only when $V^{0}=\{u\}$ and $G=H_{\left\lceil\frac{n-1}{2}\right\rceil,\left\lfloor\frac{n-1}{2}\right\rfloor}^{b, \Delta-b}$ for some positive integer $b$; if the extremal graph $G$ belongs to Case 1, then by inequalities (2.2) we have

$$
\lambda_{\min }(G) \leq-\sqrt{\left\lceil\frac{n-1}{2}\right\rceil\left\lfloor\frac{n-1}{2}\right\rfloor},
$$

where equality holds if and only if $\Delta=n-1$ is even. Thus, the extremal graph $G$ belongs to Case 1 and the desired results (i) and (ii) follow from the Case 1.

3. Proof of Corollary 1.3. (i) If $n$ is even, then let $n=2 k$. From the proof of (i) and Eq. (2.1), it suffices to show

$$
1<\frac{(n-1-\Delta)(n-1-\Delta-2 \lambda)}{2\left[(\lambda+1)^{2}-n+\Delta\right]} \leq \frac{3}{2}
$$

for $-\sqrt{\left\lceil\frac{n}{2}\right\rceil\left\lfloor\frac{n}{2}\right\rfloor} \leq \lambda \leq-\sqrt{\left\lceil\frac{n-1}{2}\right\rceil\left\lfloor\frac{n-1}{2}\right\rfloor}$ and $\left\lceil\frac{n}{2}\right\rceil+1<\Delta \leq \frac{3 n-2-\sqrt{3 n^{2}-12 n}}{2}$. In view of Claim 2.1. we only need to show that the lower bound holds, which is equivalent to

$$
2 \lambda^{2}+2 \lambda(n+1-\Delta)+2(1+\Delta-n)-(n-1-\Delta)^{2}<0 .
$$

Thus, it follows from

$$
\begin{aligned}
& 2 \lambda^{2}+2 \lambda(n+1-\Delta)+2(1+\Delta-n)-(n-1-\Delta)^{2} \\
\leq & 2 k(k-1)-2 \sqrt{k(k-1)}(2 k-\Delta+1)+2(1+\Delta-2 k)-(2 k-1-\Delta)^{2} \\
< & 2 k(k-1)-2(k-1)(2 k-\Delta+1)+2(1+\Delta-2 k)-(2 k-1-\Delta)^{2} \\
= & -\Delta^{2}+2(3 k-1) \Delta-6 k^{2}-1 \\
\leq & 0,
\end{aligned}
$$

for $\Delta \leq 3 k-1-\sqrt{3 k^{2}-6 k}=\frac{3 n-2-\sqrt{3 n^{2}-12 n}}{2}$.

If $n$ is odd, then let $n=2 k+1$. In view of the proof of (i) and Eq. (2.1), we know that it suffices to show

$$
\frac{1}{2}<\frac{(n-1-\Delta)(n-1-\Delta-2 \lambda)}{2\left[(\lambda+1)^{2}-n+\Delta\right]}<\frac{3}{2}
$$

for $-\sqrt{\left\lceil\frac{n}{2}\right\rceil\left\lfloor\frac{n}{2}\right\rfloor} \leq \lambda \leq-\sqrt{\left\lceil\frac{n-1}{2}\right\rceil\left\lfloor\frac{n-1}{2}\right\rfloor}$ and $\left\lceil\frac{n}{2}\right\rceil+1<\Delta \leq \frac{3 n-1-\sqrt{2 n^{2}-2 n+1}}{2}$. Thus, we only need to show that the lower bound holds, that is

$$
\lambda^{2}+2(2 k-\Delta+1) \lambda-2 k+\Delta-(2 k-\Delta)^{2}<0 .
$$


Hence, it follows from

$$
\begin{aligned}
& \lambda^{2}+2(2 k-\Delta+1) \lambda-2 k+\Delta-(2 k-\Delta)^{2} \\
< & k(k+1)-2(2 k-\Delta+1) k-2 k+\Delta-(2 k-\Delta)^{2} \\
= & -\Delta^{2}+(1+6 k) \Delta-7 k^{2}-3 k \\
\leq & 0
\end{aligned}
$$

for $\Delta \leq \frac{6 k+1-\sqrt{8 k^{2}+1}}{2}=\frac{3 n-2-\sqrt{2 n^{2}-4 n+3}}{2}$.

(ii) Note that it follows from the proof of (i) for $\Delta=n-1$. Thus, in what follows we only need to consider the case for $\Delta \leq n-2$.

If $n$ is even, then let $n=2 k$. From the proof of (i) and Eq. (2.1), it suffices to show

$$
0<\frac{(n-1-\Delta)(n-1-\Delta-2 \lambda)}{2\left[(\lambda+1)^{2}-n+\Delta\right]}<1
$$

for $-\sqrt{\left\lceil\frac{n}{2}\right\rceil\left\lfloor\frac{n}{2}\right\rfloor} \leq \lambda \leq-\sqrt{\left\lceil\frac{n-1}{2}\right\rceil\left\lfloor\frac{n-1}{2}\right\rfloor}$ and $\left\lceil\frac{3 n-\sqrt{3 n^{2}-8 n+4}}{2}\right\rceil \leq \Delta \leq n-2$. Consequently, we only need to show that the upper bound holds, which follows from

$$
\begin{aligned}
& 2 \lambda^{2}+2 \lambda(n+1-\Delta)+2(1+\Delta-n)-(n-1-\Delta)^{2} \\
> & 2 k(k-1)-2 k(2 k-\Delta+1)+2(1+\Delta-2 k)-(2 k-1-\Delta)^{2} \\
= & -\Delta^{2}+6 k \Delta-6 k^{2}-4 k+1 \\
\geq & 0
\end{aligned}
$$

for $\frac{3 n-\sqrt{3 n^{2}-8 n+4}}{2}=3 k-\sqrt{3 k^{2}-4 k+1} \leq \Delta \leq n-2$.

If $n$ is odd, then let $n=2 k+1$. From the proof of (i) and Eq. (2.1), it suffices to show

$$
0 \leq \frac{(n-1-\Delta)(n-1-\Delta-2 \lambda)}{2\left[(\lambda+1)^{2}-n+\Delta\right]}<\frac{1}{2}
$$

for $-\sqrt{\left\lceil\frac{n}{2}\right\rceil\left\lfloor\frac{n}{2}\right\rfloor} \leq \lambda \leq-\sqrt{\left\lceil\frac{n-1}{2}\right\rceil\left\lfloor\frac{n-1}{2}\right\rfloor}$ and $\frac{3 n-2-\sqrt{2 n^{2}-6 n+5}}{2} \leq \Delta \leq n-2$. Consequently, we only need to show that the upper bound holds, which follows from

$$
\begin{aligned}
& \lambda^{2}+2(2 k-\Delta+1) \lambda-2 k+\Delta-(2 k-\Delta)^{2} \\
> & k^{2}-2(2 k-\Delta+1) k-2 k+\Delta-(2 k-\Delta)^{2} \\
= & -\Delta^{2}+3(1+2 k) \Delta-7 k^{2}-4 k \\
\geq & 0
\end{aligned}
$$

for $\frac{3 n-2-\sqrt{2 n^{2}-6 n+5}}{2}=\frac{6 k+1-\sqrt{8 k^{2}-4 k+1}}{2} \leq \Delta \leq n-2$. 
Acknowledgment. The author is very grateful to the referee for his/her valuable suggestions towards improving this paper.

\section{REFERENCES}

[1] F.K. Bell, D. Cvetković, P. Rowlinson, and S.K. Simić. Graphs for which the least eigenvalue is minimal, I. Linear Algebra Appl., 429:234-241, 2008.

[2] F.K. Bell, D. Cvetković, P. Rowlinson, and S.K. Simić. Graphs for which the least eigenvalue is minimal, II. Linear Algebra Appl., 429:2168-2179, 2008.

[3] J.A. Bondy and U.S.R. Murty. Graph Theory with Applications. Macmillan Press, New York, 1976.

[4] S.M. Cioaba, D.A. Gregory, and V. Nikiforov. Extreme eigenvalues of nonregular graphs. J. Combin. Theory Ser. B, 97(3):483-486, 2007.

[5] G. Constantine. Lower bounds on the spectra of symmetric matrices with nonnegative entries. Linear Algebra Appl., 65:171-178, 1985.

[6] D. Cvetković, M. Doob, and H. Sachs. Spectra of Graphs, third edition. Johann Ambrosius Barth Verlag, Heidelberg, Leipzig, 1995.

[7] Y.-Z. Fan, Y. Wang, and Y.-B. Gao. Minimizing the least eigenvalues of unicyclic graphs with application to spectral spread. Linear Algebra Appl., 429:577-588, 2008.

[8] R. Liu, M. Zhai, and J. Shu. The least eigenvalues of unicyclic graphs with $n$ vertices and $k$ pendant vertices. Linear Algebra Appl., 431:657-665, 2009.

[9] M. Petrović, B. Borovićanin, and T. Aleksić. Bicyclic graphs for which the least eigenvalue is minimum. Linear Algebra Appl., 430(4):1328-1335, 2009.

[10] Y. Wang and Y.-Z. Fan. The least eigenvalue of a graph with cut vertices. Linear Algebra Appl., 433:19-27, 2010.

[11] M.-L. Ye, Y.-Z. Fan, and D. Liang. The least eigenvalue of graphs with given connectivity. Linear Algebra Appl., 430(4):1375-1379, 2009.

[12] B.-X. Zhu. Bounds on the eigenvalues of graphs with cut vertices or edges. Linear Algebra Appl., 434:2030-2041, 2011. 